\title{
On the accuracy of $N-1$ wire-driven parallel robots
}

\author{
Jean-Pierre Merlet* \\ INRIA Sophia-Antipolis
}

\begin{abstract}
A $N-1$ wire-driven parallel robot is a robot for which all the $N \geq 3$ wires are connected at the same point of the platform, allowing to control the location of this point. We are interested in the positioning accuracy of such a robot. If the wires are not elastic we show that the influence on the accuracy of the co-location errors of the wire anchor points on the platform is moderate, although a full analysis is a very difficult task. If the wires are elastic we study the influence of the the wire lengths measurement errors and inaccurate estimation of the stiffness of the wires. Again we show a moderate influence but very large changes in the tensions in the wires that probably prohibit the use of the redundancy to optimize the tension in the wires. In all cases the complexity of the forward kinematics of such a robot makes accuracy analysis a very demanding task that requires an in-depth investigation.
\end{abstract}

\section{The $N-1$ wire-driven parallel robot}

In a wire-driven parallel robot (WDPR) wires are attached at specific anchor points on the robot platform and can be coiled and uncoiled through an actuation system with a fixed output point for the wires. WDPR have been introduced in the 80's (Landsberger and Sheridan, 1985),(Miura and Furuya, 1984) as an alternate to parallel robot with rigid links. They share with them the ability to manipulate large load and to be energy efficient ( $\mathrm{Li}$ and Bone, 2001) while they allow for larger workspace (as the amount of leg lengths variation is much larger) and present a simpler mechanical design. However their major drawback is that wires can be pulled but not pushed, which increases the complexity of their kinematics as statics has to be taken into account (for example the forward kinematic problem is an open issue (Carricato and Merlet, 2011)).

\footnotetext{
${ }^{*}$ The author acknowledges the partial support of the EU through the grant 285404 CableBOT CP-FP
} 
There has been recently a renewal of interest for WDPR in view of new applications: wind tunnel (Yaqing et al., 2007), biomechanic and rehabilitation (Wu et al., 2011), haptic interface (V. Zitzewitz et al., 2009), rescue robotics (Takeda et al., 2005),(Merlet and Daney, 2010). However an important point has not been addressed completely in this field: positioning accuracy. This is a very well addressed field for parallel robots with rigid links (Merlet, 2006) but still an open issue for WDPR whose kinematics is much more complex (Ottaviano et al. (2002); Thomas et al. (2002)).

We will address this problem for a specific class of WDPR, the $N-1$ WDPR which has $N$ wires attached at the same point on the platform, allowing to control the location of this point but not the platform orientation. We will consider two different cases for the wires: non-elastic and elastic.

\section{Non-elastic wires}

Although this is not the scope of this paper, an important result has to be presented if $N>3$ :

At any pose a $N-1$ robot with non-elastic wires will have at most 3 wires under tension whatever is $N>3$

This new result, that will be presented at ICRA 2012, is important as it allows to reduce the accuracy analysis of a $N-1$ robot to the accuracy analysis of the four 3-1 robots that are derived from the initial robot.

The exit point of the wire system $i$ will be denoted by $A_{i}$, its wire length as $\rho_{i}$ and the tension in the wire as $\tau_{i}$. The platform pose is determined by the coordinates $x, y, z$ of $C$, the center of mass of the load, in a reference frame where the $\mathbf{z}$ axis is vertical. The anchor points of the wires will be denoted by $B_{i}$ (ideally $B_{i}=C$ ). Note that the inverse kinematics (IK) is straightforward as we have

$$
\rho_{i}^{2}=\left\|\mathbf{A}_{\mathbf{i}} \mathbf{B}_{\mathbf{i}}\right\|^{2}
$$

Let us assume that the platform is submitted to a force $\mathcal{F}$. The relation between this force and the tension in the wires is given by:

$$
\mathcal{F}=\mathbf{J}^{-T} \tau
$$

where $\mathbf{J}^{-T}$ is the transpose of the inverse jacobian matrix of the robot. The ith column $J_{i}^{-T}$ of this matrix is: $J_{i}^{-T}=\mathbf{A}_{\mathbf{i}} \mathbf{C}^{T} / \rho_{i}$. Note that with this convention wire $i$ is under tension if $\tau_{i}$ is positive. For this robot the sources of inaccuracy are errors in the wire lengths, in the location of the exit points of the wire systems and in the location of the common attachment point. Influence of the wire lengths errors is a well studied topics (Murphy, 
2007),(Thomas et al., 2002) and will not be considered here. We will also assume that the location of the $A_{i}$ are well known . Hence remains possible errors on the location of the $B_{i}$.

\subsection{Three distinct attachment points $B_{i}$}

We will first assume that the 3 wires are attached at three distinct points on the platform, that are close to $C$ but distinct from it. To study the accuracy of the robot we will assume a given position of $C$ and we will calculate the wire lengths with equation (1). Then we will assume that the $B_{i}$ are different from $C$ (which implies that the wire lengths affect the orientation of the platform) and solve the forward kinematics (FK). The difference between the obtained pose and the theoretical one will give us the positioning error. Unfortunately we are confronted to a major issue: the FK for a 3-3 robot is still an open problem. For the FK we have as unknowns the 6 parameters of the pose of the platform and the 3 tensions in the wires while we have 3 kinematics equations (1) and 6 statics equations (2). It has been shown that the solution(s) of this system may be calculated in theory by solving a 158th order univariate polynomial (Carricato and Merlet, 2011) but the high order of this polynomial makes the solving quite difficult. In our case we rely however on an alternate approach based on interval analysis. As an example we will consider the 3 - 1 robot with $A_{1}(0,0,0), A_{2}(400,0,0), A_{3}(0,400,0)$ and consider that the $B_{i}$ lie on a circle of radius 5 so that $C B_{1}(-5,0,0), C B_{2}(-2.5,2.5 \sqrt{3}, 0), C B_{3}(2.5,2.5 \sqrt{3}, 0)$.

For $C$ defined by $(176.375,192.375,-147.93)$ the theoretical wire lengths are $300,310,330$ and the FK admits two solutions with positive tensions: $(171.72,187.24,-152.66)$ with the Euler angles in radian $(-0.727,-0.387$, $1.62)$ and $(178.465,194.68,-163.99)$ with the angles $(-0.76,0.324,1.55)$. We are confronted here with a difficulty of the FK of WDPR that may admit several solutions, this increasing the complexity of the accuracy analysis. However we note the moderate positioning errors with a distance between the solutions and the theoretical one of 8.39 and 16.36 . If the radius of the circle for the $B_{i}$ 's is reduced to 1 , then we still get 2 solutions at a distance 1.99 and 2.0 from the theoretical one.

\subsection{Attachment points $B_{i}$ on a common ring}

We will consider here that the 3 wires are connected to a ring of center $U$ and radius $r$ and are free to slide on this ring, although their motion must respect their initial connecting order. We will assume that the plane that includes the ring is perpendicular to the platform and that $U, C$ lie on the same normal to the platform (figure 1). 


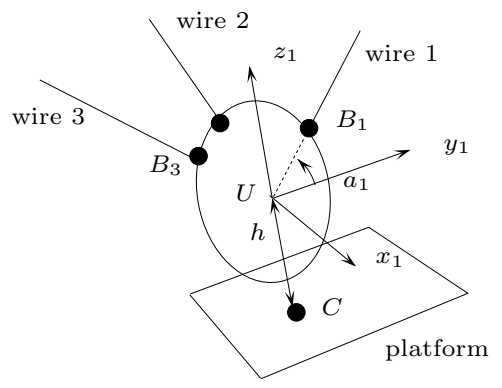

Figure 1. The wires may slide on a ring that is attached to the platform

We define a frame $\mathcal{F}_{1}=U, \mathbf{x}_{1}, \mathbf{y}_{1}, \mathbf{z}_{1}$ attached to the ring so that $\mathbf{x}_{1}$ is perpendicular to the ring plane. In this frame the coordinates of $B_{i}$ are

$$
\mathbf{U B}_{\mathbf{i}}{ }^{1}=\left(0, r \cos \left(a_{i}\right), r \sin \left(a_{i}\right)\right)
$$

where $a_{i}$ is the angle between $\mathbf{U B}_{\mathbf{i}}$ and the $\mathbf{y}_{1}$ axis. We then define the mobile frame of the platform $\mathcal{F}_{r}=\left(C, \mathbf{x}_{\mathbf{r}}, \mathbf{y}_{\mathbf{r}}, \mathbf{z}_{\mathbf{r}}\right)$ so that $\mathbf{z}_{\mathbf{r}}$ is parallel to $\mathbf{z}_{\mathbf{1}}$. Hence the coordinates in $\mathcal{F}_{r}$ of a vector $\mathbf{u}$ whose coordinates are known in $\mathcal{F}_{1}$ are obtained as $\mathbf{R}_{1} \mathbf{u}$ where $\mathbf{R}_{1}$ is the rotation matrix for a rotation around the axis $\mathbf{z}_{r}$ of angle $\psi_{1}$. As $U$ lies on the $\mathbf{z}_{r}$ axis we have $\mathbf{C} U_{r}=(0,0, h)$. If we define $\mathbf{R}$ as the rotation matrix between the reference frame and $\mathcal{F}_{r}$ we get:

$$
\mathbf{C B}_{\mathbf{i}}=\mathbf{R C B}_{\mathbf{i}}^{\mathbf{r}}=\mathbf{R}\left(\mathbf{C U}_{\mathbf{r}}+\mathbf{R}_{1} \mathbf{U B}_{\mathbf{i}}{ }^{1}\right)
$$

while

$$
\mathbf{A}_{\mathbf{i}} \mathbf{B}_{\mathbf{i}}=\mathbf{A}_{\mathbf{i}} \mathbf{O}+\mathbf{O C}+\mathbf{C B}_{\mathbf{i}}
$$

Equation (2) is now a linear system of 6 equations in the 3 unknowns $\tau_{i}$, which is dependent upon the 3 unknowns $a_{i}$ and the 6 pose parameters. Three of these equations may be used to obtain the $\tau_{i}$ as functions of the $a_{i}$ and of the pose parameters and will remain three constraint equations.

If we assume now that there is no contact between the attachment points $B_{i}$ of the wires on the ring we write that at equilibrium the force exerted by the wires at $T_{i}$ must be perpendicular to the ring tangent at $B_{i}$. In the frame $\mathcal{F}_{1}$ the ring tangent vector $\mathbf{T}_{i}{ }^{1}$ is

$$
\mathbf{T}_{i}{ }^{1}=\left(0,-r \sin \left(a_{i}\right), r \cos \left(a_{i}\right)\right)
$$

Hence in the reference frame we have $\mathbf{T}_{i}=\mathbf{R} R_{1} \mathbf{T}_{i}{ }^{1}$. We get thus 3 additional constraint equations with

$$
\mathbf{A}_{\mathbf{i}} \mathbf{B}_{\mathbf{i}} \cdot \mathbf{T}_{\mathbf{i}}=0
$$


For the FK problem if we assume that all the $B_{i}$ are distinct, then we have as unknowns the 6 pose parameters of the platform and the $3 a_{i}$. We have also 3 equations (1), 3 remaining equations from the statics (2) and the 3 constraint equations (3). Solving such a system is quite difficult and furthermore not all solutions are valid: indeed the initial assembly of the robot imposes an ordering of the wires on the ring and this ordering must be respected for the FK solution. For solving this problem we use interval analysis which has the advantage of easily allowing to enforce the ordering constraint. For the same robot than in the previous section we have considered the pose for $C$ as $(92.95,110.85,-198.02)$, which lead to the theoretical wire lengths $(237.23,376.72,357.206), r=5, h=10$ and the ordering $(2,3,1)$ for the wires on the ring. The solving leads to 2 solutions for $C,(110,100,200)$ and $(110.84,92.78,-204.22)$, with here again a relatively moderate difference between the theoretical pose and the final one. Note that the solving is computer intensive as the computation time is over $1 \mathrm{~h}$ : it appears that the influence of errors on the location of the attachment points on the platform on accuracy is a very demanding task.

\section{Elastic wires}

In this section we assume that the wires are perfect linear springs. Let $\tau_{i}$ be the tension in wire $i, l_{i}$ its length at rest, $k_{i}$ the wire stiffness and $\rho_{i}$ its length when under tension. We have

$$
\tau_{i}=k_{i}\left(\rho_{i}-l_{i}\right)
$$

Let us consider a 4-1 WDPR and its inverse kinematics. For a given pose of the load the values of the $\rho_{i}$ may be determined with equation (1). Equation (2) is a linear system of 3 equations in the $\tau_{i}$ that allows one to calculate $\tau_{2}, \tau_{3}, \tau_{4}$ as functions of $\tau_{1}$. For a given value of $\tau_{1}$ we may compute the remaining $\tau_{i}$ and, if all the $\tau_{i}$ are positive, we get the value of $l_{i}$ from equation (4). The choice of $\tau_{1}$ is free and hence we have a redundant robot that allows, in theory, to manage the wires tensions distribution. We may choose, for example, a $\tau_{1}$ such that $H=\sum_{j=1}^{j=4} \tau_{j}^{2}$ is minimized. This function is quadratic in $\tau_{1}$ and hence finding the optimal $\tau_{1}$ is trivial. Note however that we may have IK solutions such that not all 4 wires are under tension.

For the accuracy analysis we have to solve the FK problem. Here the $l_{i}$ are given and the pose of the load has to be determined. The first equation of (4) allows one to determine $\tau_{1}$. Equation (2) is used to determine the values of $\tau_{2}, \tau_{3}, \tau_{4}$ as functions of $\tau_{1}$. The three remaining equations of (4) are linear in the coordinates of $C$. After solving this system we report the result in the IK equations (1) which constitutes a system of 4 equations 
in the unknowns $\rho_{1}, \rho_{2}, \rho_{3}, \rho_{4}$. The difference between the first and second equation is linear in $\rho_{4}$ and is solved for this variable. The 3 remaining equations, denoted $a_{1}, a_{2}, a_{3}$, are of degree $(6,6,2),(3,3,3),(9,9,3)$ in $\rho_{1}, \rho_{2}, \rho_{3}$. Successive resultants between these equations leads to a polynomial in $\rho_{1}$ only, which factors out in 2 polynomials of degree 76 and 96 . Although this complete the theoretical solution the degree of the involved polynomials are too high to be used in practice and consequently we have to resort to a numerical procedure. For that purpose we solve the linear system (2) to get $\tau_{2}, \tau_{3}, \tau_{4}$ as function of $\tau_{1}$. Then the first equation of (4) is used to determine $\tau_{1}$ as a function of $\rho_{1}$. The three remaining equations of (4) together with the 4 equations of (1) constitutes a system of 7 equations in the 7 unknowns $x, y, z, \rho_{1}, \rho_{2}, \rho_{3}, \rho_{4}$, which is solved using interval analysis. However we have also to consider that this system may not have a solution as in the final configuration less than 4 wires may be under tension. If 3 wires are under tension equation (2) is solved to determine the $\tau_{i}$, the result being reported in equations (4) to obtain 3 constraint equations in $x, y, z$ and the $3 \rho_{i}$. With the 3 equations (1) we get a system of 6 equations in the 6 unknowns. As we have to consider all combinations of 3 wires among 4 , we have to solve four such system.

To test the sensitivity of the solving to uncertainties on the $l_{i}$ 's and on $k_{i}$ we have considered the 4 - 1 robot with $A_{1}(0,0,0), A_{2}(400,0,0), A_{3}(0,400,0)$, $A_{4}(400,400,0)$ and we have used the IK to determine what should be the $l_{i}$ to reach the pose $x=100, y=200, z=-200$ with a load of 80 , while minimizing $\sum_{j=1}^{j=4} \tau_{i}^{2}$, assuming an identical stiffness $k=1000$ for all wires. The nominal values for the $l_{i}$ are $l_{1}=l_{2}=299.558, l_{3}=l_{4}=412.108$ which leads to $\tau_{1}=\tau_{2}=441.45, \tau_{3}=\tau_{4}=202.238$. We have then considered 1000 values for the $k_{i}$ that were randomly perturbed around their nominal values by $\pm 0.1 k$. We have then calculated the FK by assuming first that all 4 wires were under tension and then the FK with only 3 wires under tension (all combination of 3 wires were considered) and assuming a perfect wire lengths control. For all 1000 tests we have obtained a single solution with 4 wires under tension with the ranges [99.95,100.045], [199.94, 200.05], [-200.054,199.95] for $x, y, z$. However we have observed large variations in $\tau_{i}$, that lie in the ranges [421.3, 462], [420.8, 461.5], [174.1,229.7],[174.7,230.4]. Furthermore there was always a single solution with wires $(1,2,3)$ and $(1,2,4)$ under tension, while the remaining wire is slack. For these solutions the ranges for $x, y, z$ were [99.61,99.74], [199.71,200.28],[-200.23,-200.09]. In the triplet $(1,2,3)$ the tension in wire 3 is almost constant (range: [402.78,403.35]) as it is for the tension in wire 4 for the triplet $(1,2,4)$ (range: $[402.78,403.35]$ ) but the tension in wires 1,2 were changing significantly for the two triplets with typical values of $(295,588)$ for the first triplet and $(588,295)$ for the 
second one. Hence force measurements will allow to determine if the wires under tension are $(1,2,3,4),(1,2,3)$ or $(1,2,4)$.

We then perturb both $l_{i}$ (with a range of \pm 3 ) and $k_{i}$. In that case for 1000 tests, only 161 where admitting a solution with 4 wires under tension with the ranges $[95.62,103.97]$ for $x$ (mean value:100.18), [196.31,203.17] for $y$ (mean value:200), [-204.22,-195.67] for $z$ (mean value:-200.13), with large variations for the $\tau_{i}$ (ranges: [289.4,583.15], [290.8,590.45],[7.7, 407.2], [0.8, 402.4]). Here again there was always at least 2 solutions with 3 wires under tension with a range [94.5,104.8] for $x$ (mean value: 99.7), [195.54,204.72] for $y$ (mean value: 199.97), [-204.52,-195.72] for $z$ (mean value: -200.25). For the triplet $(1,2,3) \tau_{3}$ lies in the range [381.5, 426.5] while for the triplet $(1,2,4) \tau_{4}$ lies in the range $[383.8,425.6]$. Still force measurements allows one to determine the configuration of wire under tension as $\tau_{1}$ either lies in the range $[273.56,315.3]$ or $[574.8,602.15]$ while $\tau_{2}$ lies in the range $[274.5,313.96]$ or $[573.9,603]$. Similar results were obtained for $k=10$ and $k=100$.

In conclusion positioning errors are moderate, while the variations of wire tensions probably prohibit the use of force control and the use of the redundancy to manage distribution of the tension in the wires.

\section{Conclusion}

Although WDPR have attracted a lot of interest recently there has been few works that address their positioning accuracy. We have considered a specific class of WDPR for which all wires are assumed to be attached at the same point on the platform. For non elastic wires the main source of positioning errors (beside control errors in the wire lengths) is that in practice the wires are not connected at the same point. We have shown that finding the pose of the robot when assuming close but distinct attachment points is a difficult task and seems to lead to moderate positioning errors. We have then considered WDPR with elastic wires and have shown that errors on the stiffness of the wires and/or on the wire lengths may also lead to moderate positioning errors but large variations in the wire tensions.

Accuracy analysis of WDPR is a complex task because we have to consider the FK problem for all possible combinations of wires under tension (and not only the case where all $N$ wires are under tension), while many of these problems are open. For the $N-1$ WDPR we have shown that the positioning errors seem to be moderate but that there is large variations of wire tensions, which probably prohibit the use of force control and redundancy management. Hence this issue requires still an in-depth investigation. 


\section{Bibliography}

M. Carricato and J-P. Merlet. Direct geometrico-static problem of underconstrained cable-driven parallel robots with three cables. In IEEE Int. Conf. on Robotics and Automation, pages 3011-3017, Shangai, May, 9-13, 2011.

S.E. Landsberger and T.B. Sheridan. A new design for parallel link manipulator. In Proc. Systems, Man and Cybernetics Conf., pages 812-814, Tucson, 1985.

Y. Li and G.M. Bone. Are parallel manipulators more energy efficient ? In IEEE Int. Symp. on Computational Intelligence in Robotics and Automation, Banff, August 29- September 1, 2001.

J-P. Merlet. Computing the worst case accuracy of a PKM over a workspace or a trajectory. In 5th Chemnitzer Parallelkinematik Seminar, pages 8396, Chemnitz, April, 25-26, 2006.

J-P. Merlet and D. Daney. A portable, modular parallel wire crane for rescue operations. In IEEE Int. Conf. on Robotics and Automation, pages 2834-2839, Anchorage, May, 3-8, 2010.

K. Miura and H. Furuya. Variable geometry truss and its application to deployable truss and space crane arms. In 35th Congress of the Int. Astronautical Federation, pages 1-9, Lausanne, October, 7-13, 1984.

W.S. Murphy. Determination of a position using approximate distances and trilateration. Master's thesis, Colorado School of Mines, Golden, July 2007.

E. Ottaviano et al. CaTraSys (Cassino Traking System): A wire system for experimental evaluation of robot workspace. Robotics and Mechatronics, 14(1):78-87, 2002.

Y. Takeda et al. A human body searching strategy using a cable-driven robot with an electromagnetic wave direction finder at major disasters. Advanced Robotics, 19(3):331-347, 2005.

F. Thomas et al. Uncertainty model and singularities of 3-2-1 wire-based tracking systems. In $A R K$, pages 107-116, Caldes de Malavalla, June 29- July 2, 2002.

J. V. Zitzewitz et al. A versatile wire robot concept as a haptic interface for sport simulation. In IEEE Int. Conf. on Robotics and Automation, pages 313-318, Kobe, May, 14-16, 2009.

$\mathrm{M}$. Wu et al. A cable-driven locomotor training system for restoration of gait in human SCI. Gait \& Posture, 33(2):256-260, February 2011.

Z. Yaqing, L. Qi, and L. Xiongwei. Initial test of a wire-driven parallel suspension system for low speed wind tunnels. In 12th IFToMM World Congress on the Theory of Machines and Mechanisms, Besancon, June, 18-21, 2007. 